Commun.Fac.Sci.Univ.Ank.Series A 1

Volume 56, Number 2, Pages 17-25 (2007)

ISSN $1303-5991$

\title{
SOME BOUNDS FOR THE $n$-FOLD CONVOLUTION OF CONCAVE AND LOG-CONCAVE DISTRIBUTION FUNCTIONS
}

\author{
HALIL AYDOĞDU
}

\begin{abstract}
In general it is impossible to obtain analytical expressions for the $n$-fold convolution $F^{n *}$ of a distribution function $F$. Existence of bounds for $F^{n *}$ is of great value. In this study some bounds for $F^{n *}$ are given with the help of the probability integral transformation when $F$ is concave or log-concave.
\end{abstract}

\section{INTRODUCTION}

The $n$-fold convolution of a distribution function is of great interest in applied probability theory. Many expressions in reliability and renewal theory involve this convolution.

Let $F$ and $G$ be any distribution functions. The function $F * G$ defined as

$$
F * G(t)=\int_{-\infty}^{\infty} G(t-x) d F(x), \quad t \in R,
$$

is called the convolution of $F$ and $G$. The convolution $F * G$ is again a distribution function. We write $F^{2 *}$ for $F * F$. For any $n \in N$, the $n$-fold convolution of $F$ with itself is defined as

$$
F^{n *}(t)= \begin{cases}F(t), & n=1 \\ \int_{-\infty}^{\infty} F^{(n-1) *}(t-x) d F(x), & n>1 .\end{cases}
$$

The support of $F$ for the distributions arising most commonly in practice is $[0, \infty)$. Then, for $n>1$,

$$
F^{n *}(t)=\int_{0}^{\infty} F^{(n-1) *}(t-x) d F(x)=\int_{0}^{t} F^{(n-1) *}(t-x) d F(x) .
$$

Received by the editors Octb.30, 2007; Accepted: Dec.14, 2007.

2000 Mathematics Subject Classification.

Key words and phrases. Convolution, concave df, log-concave df. 
It is well known that $F^{n *}$ is the distribution function of the sum of $n$ independent random variables each of which has the same distribution function $F$; that is,

$$
F^{n *}(t)=P\left(X_{1}+X_{2}+\ldots+X_{n} \leq t\right), t \in R,
$$

where $X_{1}, X_{2}, \ldots, X_{n}$ are independent and identically distributed random variables with distribution function $F$.

For some distribution functions, such as the uniform, shifted exponential, truncated exponential and Erlang (gamma with integer shape parameter), the $F^{n *}$ can be obtained in analytical form. For example, if $F$ is a distribution function of the uniform distribution on the interval $(0, \theta)$, the well known analytical expression for $F^{n *}(t)$ is

$$
F^{n *}(t)= \begin{cases}0, & t<0 \\
\frac{1}{n ! \theta^{n}} \sum_{j=0}^{k}(-1)^{j}\left(\begin{array}{l}
n \\
j
\end{array}\right)(t-j \theta)^{n}, & k \theta \leq t \leq(k+1) \theta, \\
1, & k=0,1, \ldots, n-1 \\
& t \geq n \theta .\end{cases}
$$

However, closed forms do not exist for many distributions arising in practice. Several numerical methods are available for evaluating $F^{n *}(t), n \geq 1$ [2]. The numerical evaluation of $F^{n *}(t)$ sometimes leads to different numerical errors, which are not always easy to bound. Hence, bounds for $F^{n *}(t)$ are very useful.

In this study some bounds for $F^{n *}(t)$ are given with the help of the probability integral transformation when $F$ is a concave or log-concave distribution function with support $[0, \infty)$. For a complete presentation, we first give some definitions and some known results.

Consider a continuous distribution function $F$ with support $[0, \infty) . F$ is called concave if

$$
F(\lambda s+(1-\lambda) t) \geq \lambda F(s)+(1-\lambda) F(t)
$$

for every $s, t \geq 0$ and $0 \leq \lambda \leq 1$. It is easy to see that

$F$ is concave $\Longleftrightarrow F(t+h)-F(t) \leq F(s+h)-F(s)$ for all $t \geq s \geq 0$ and $h \geq 0$.

So, we have

$$
F \text { is concave } \Longrightarrow F \text { is subadditive. }
$$

Let $F$ be concave and $\lambda_{i} \in[0,1], i=1,2,3$ such that $\lambda_{1}+\lambda_{2}+\lambda_{3}=1$. Then, from (3) it follows that

$$
\begin{aligned}
F\left(\lambda_{1} t_{1}+\lambda_{2} t_{2}+\lambda_{3} t_{3}\right) & =F\left(\left(1-\lambda_{3}\right)\left(\frac{\lambda_{1}}{1-\lambda_{3}} t_{1}+\frac{\lambda_{2}}{1-\lambda_{3}} t_{2}\right)+\lambda_{3} t_{3}\right) \\
& \geq\left(1-\lambda_{3}\right) F\left(\frac{\lambda_{1}}{1-\lambda_{3}} t_{1}+\frac{\lambda_{2}}{1-\lambda_{3}} t_{2}\right)+\lambda_{3} F\left(t_{3}\right) \\
& \geq\left(1-\lambda_{3}\right) \frac{\lambda_{1}}{1-\lambda_{3}} F\left(t_{1}\right)+\left(1-\lambda_{3}\right) \frac{\lambda_{2}}{1-\lambda_{3}} F\left(t_{2}\right)+\lambda_{3} F\left(t_{3}\right) \\
& =\lambda_{1} F\left(t_{1}\right)+\lambda_{2} F\left(t_{2}\right)+\lambda_{3} F\left(t_{3}\right) .
\end{aligned}
$$

In general, 


$$
F \text { is concave } \Longrightarrow F\left(\lambda_{1} t_{1}+\ldots+\lambda_{n} t_{n}\right) \geq \lambda_{1} F\left(t_{1}\right)+\ldots+\lambda_{n} F\left(t_{n}\right)
$$

for $\lambda_{i} \in[0,1], i=1,2, \ldots, n$ such that $\sum_{i=1}^{n} \lambda_{i}=1$.

The distribution function $F$ is called log-concave if $\ln F$ is concave. Suppose that $F$ is absolutely continuous with probability density function $f$. If $f$ is continuously differentiable and log-concave, then $F$ is also log-concave [5]. Since the logarithm function $\ln$ is increasing and concave, it is clear that

$$
F \text { is concave } \Longrightarrow F \text { is log-concave. }
$$

However, the converse of $(6)$ is not true. For example, let $F$ be the Weibull distribution function with parameters $\alpha=2$ and $\beta>0$; that is, $F(t)=1-e^{-(t / \beta)^{2}}, t \geq 0$. The corresponding probability density function is $f(t)=\frac{2}{\beta^{2}} t e^{-(t / \beta)^{2}}, t \geq 0$. By differentiating $F(t)$ and $\ln f(t)$ twice it is obtained that $F^{\prime \prime}(t)=\frac{2}{\beta^{2}} e^{-(t / \beta)^{2}}\left(1-\frac{2}{\beta^{2}} t^{2}\right)$ and $(\ln f(t))^{\prime \prime}=-\frac{2}{\beta^{2}}-\frac{1}{t^{2}}$. Since $F^{\prime \prime}(t)>0$ for $t<\frac{\beta}{\sqrt{2}}$ and $F^{\prime \prime}(t)<0$ for $t>\frac{\beta}{\sqrt{2}}$, $F$ is not concave. The sign of $(\ln f(t))^{\prime \prime}$ is negative. Therefore $F$ is $\log$-concave.

The distribution function $F$ (or the corresponding $\bar{F}=1-F$ ) is called

(i) increasing failure rate (IFR) if $\ln \bar{F}$ is concave; that is, if for all $s>0$, $\bar{F}(s+t) / \bar{F}(t)$ is non-increasing in $t$ whenever $t \geq 0$ and $\bar{F}(t)>0$. If $F$ has a density function $f$ then this is equivalent to the condition that the failure rate $r(t) \equiv f(t) / \bar{F}(t)$ is non-decreasing in $t$ on $\{t: \bar{F}(t)>0\}$;

(ii) new better than used (NBU) if $\bar{F}(s+t) \leq \bar{F}(s) \bar{F}(t)$ for all $s, t \geq 0$;

(iii) new better than used in expectation (NBUE) if the mean $\mu$ of $F$ is finite and $\mu \geq \int_{0}^{\infty}[\bar{F}(s+t) / \bar{F}(t)] d s$ for all $t \geq 0$ such that $\bar{F}(t)>0$.

By reversing the inequalities above, the notions of decreasing failure rate (DFR), new worse than used (NWU) and new worse than used in expectation (NWUE) are defined.

It is known that the DFR-ness of $F$ is a sufficient condition for $F$ to be concave [3] and the concavity of $F$ implies that $F$ is NWUE [1]. Then, from (6) the class of log-concave distribution functions is broader than the class of DFR distribution functions. Note that some IFR distribution functions such as gamma $(\alpha \geq 1, \beta>0)$ and Weibull $(\alpha \geq 1, \beta>0)$ are log-concave.

\section{Results}

From (1) it follows that $F^{(n+1) *}(t) \leq F(t) F^{n *}(t), n=1,2, \ldots$. Then, $F^{n *}(t) \leq$ $F^{n}(t)$ for $t \geq 0$ and $n=1,2, \ldots$. Some better bounds are given by Barlow and Proschan [4]. Suppose that $F$ is continuous. They have shown that for $t \geq 0$ and $n=1,2, \ldots$,

$$
F \text { is IFR }(\mathrm{DFR}) \Longrightarrow F^{n *}(t) \geq(\leq) 1-\sum_{j=0}^{n-1} \frac{\left(n R\left(\frac{t}{n}\right)\right)^{j}}{j !} e^{-n R\left(\frac{t}{n}\right)}
$$


and

$$
F \text { is } \mathrm{NBU}(\mathrm{NWU}) \Longrightarrow F^{n *}(t) \leq(\geq) 1-\sum_{j=0}^{n-1} \frac{R^{j}(t)}{j !} e^{-R(t)}
$$

where $R$ is the hazard function; that is, $R(t)=-\ln \bar{F}(t)$.

It is well known that if $F$ is IFR (DFR) then $F$ is NBU (NWU). Therefore, from (7) and (8) it can be written that

$$
F \text { is IFR } \Longrightarrow 1-\sum_{j=0}^{n-1} \frac{\left(n R\left(\frac{t}{n}\right)\right)^{j}}{j !} e^{-n R\left(\frac{t}{n}\right)} \leq F^{n *}(t) \leq 1-\sum_{j=0}^{n-1} \frac{R^{j}(t)}{j !} e^{-R(t)}
$$

and

$$
F \text { is DFR } \Longrightarrow 1-\sum_{j=0}^{n-1} \frac{R^{j}(t)}{j !} e^{-R(t)} \leq F^{n *}(t) \leq 1-\sum_{j=0}^{n-1} \frac{\left(n R\left(\frac{t}{n}\right)\right)^{j}}{j !} e^{-n R\left(\frac{t}{n}\right)}
$$

for $t \geq 0$ and $n=1,2, \ldots$.

Now assume that $F$ is concave. We give an upper bound for $F^{n *}(t)$ as the following theorem.

Theorem 1. If $F$ is continuous and concave then for $n=1,2, \ldots$,

$$
F^{n *}(t) \leq \begin{cases}\frac{1}{n !} \sum_{j=0}^{k}(-1)^{j}\left(\begin{array}{c}
n \\
j
\end{array}\right)\left(n F\left(\frac{t}{n}\right)-j\right)^{n}, & n F^{-1}\left(\frac{k}{n}\right) \leq t<n F^{-1}\left(\frac{k+1}{n}\right), \\
& k=0,1, \ldots, n-1 \\
1, & t \geq n F^{-1}(1),\end{cases}
$$

where $F^{-1}(0)=0$ and $F^{-1}(t)=\inf \{x: F(x) \geq t\}, 0<t \leq 1$.

Proof. It is clear that for $t \geq 0$ and $n=1,2, \ldots$,

$$
\begin{aligned}
F^{n *}(t) & =P\left(X_{1}+X_{2}+\ldots+X_{n} \leq t\right) \\
& =P\left(F\left(\frac{X_{1}+X_{2}+\ldots+X_{n}}{n}\right) \leq F\left(\frac{t}{n}\right)\right),
\end{aligned}
$$

where $X_{1}, X_{2}, \ldots, X_{n}$ are independent and identically distributed random variables with distribution function $F$. Using the concavity of $F$ with $\lambda_{i}=\frac{1}{n}, i=1,2, \ldots, n$ in (5) we have

$$
F\left(\frac{X_{1}+X_{2}+\ldots+X_{n}}{n}\right) \geq \frac{F\left(X_{1}\right)+F\left(X_{2}\right)+\ldots+F\left(X_{n}\right)}{n} .
$$

$F\left(X_{i}\right)(i=1,2, \ldots, n)$ are independent and identically distributed random variables. Then, it follows from the above inequality and the probability integral transformation that

$$
\begin{aligned}
F^{n *}(t) & \leq P\left(F\left(X_{1}\right)+F\left(X_{2}\right)+\ldots+F\left(X_{n}\right) \leq n F\left(\frac{t}{n}\right)\right) \\
& =F_{U}^{n *}\left(n F\left(\frac{t}{n}\right)\right)
\end{aligned}
$$


where $U$ is a uniform random variable on $(0,1)$. Hence, from the analytical expression with $\theta=1$ in (2) the inequality (11) is obtained. Thus the proof is completed.

Note that we take $F^{-1}(1)=\infty$ in $(11)$ if $F(t)<1$ for all $t$.

Example 1. Let $F$ be the uniform distribution function on the interval $(0, \theta)$. This distribution function is both concave and IFR. It is obvious that the upper bound in (11) is equal to $F^{n *}(t)$ given in (2) for all $t \geq 0$ and $n=1,2, \ldots$. Thus, this upper bound is the best for the uniform distribution on $(0, \theta)$.

Example 2. Consider the following distribution function

$$
F(t)= \begin{cases}0, & t<0 \\ t-\frac{t^{2}}{4}, & 0 \leq t<2 \\ 1, & t \geq 2\end{cases}
$$

It is clear that $F$ is concave. Since the failure rate function $r(t)=(4-2 t) /(4-4 t+$ $\left.t^{2}\right), 0 \leq t<2$ is increasing, $F$ is also IFR. Then, the upper bound in Theorem 1 or (9) given by Barlow and Proschan [4] can be used for $F^{n *}(t)$. Let us take $n=2$. Then we obtain

$$
F^{2 *}(t)= \begin{cases}0, & t<0 \\ \frac{t^{2}}{2}-\frac{t^{3}}{6}+\frac{t^{4}}{96}, & 0 \leq t<2 \\ -\frac{5}{3}+\frac{8 t}{3}-t^{2}+\frac{t^{3}}{6}-\frac{t^{4}}{96}, & 2 \leq t<4 \\ 1, & t \geq 4 .\end{cases}
$$

Let $u_{1}(t)$ and $u_{2}(t)$ denote the upper bounds in (11) and (9), respectively. We have

$$
u_{1}(t)= \begin{cases}\frac{t^{2}}{2}-\frac{t^{3}}{8}+\frac{t^{4}}{128}, & 0 \leq t \leq 1.1716 \\ -1+2 t-\frac{3 t^{2}}{4}+\frac{t^{3}}{8}-\frac{t^{4}}{128}, & 1.1716 \leq t \leq 4 \\ 1, & t \geq 4\end{cases}
$$

and

$$
u_{2}(t)= \begin{cases}t-\frac{t^{2}}{4}+\left(1-t+\frac{t^{2}}{4}\right) \ln \left(1-t+\frac{t^{2}}{4}\right), & 0 \leq t<2 \\ 1, & t \geq 2 .\end{cases}
$$

The graphs of $F^{2 *}(t), u_{1}(t)$ and $u_{2}(t)$ are given in Figure 1 . It is clear that the upper bound in Theorem 1 is better than the upper bound in (9) given by Barlow and Proschan [4].

Example 3. Let

$$
F(t)= \begin{cases}0, & t<0 \\ \frac{1-e^{-\sqrt{t}}}{1-e^{-2}}, & 0 \leq t<4 \\ 1, & t \geq 4\end{cases}
$$

In fact, this distribution is obtained by truncating the Weibull distribution with parameters $\alpha=1 / 2$ and $\beta=1$ at $t=4 . F$ is concave. Since the failure rate function $r(t)=\frac{1}{2 \sqrt{t}\left(1-e^{\sqrt{t}-2}\right)}, 0<t<4$ is not monotone, $F$ is neither IFR nor DFR. It is easy to see that if there exists a $t \in R$ such that $F(t)=1$ for any distribution function $F$, then $F$ can not be NWU. Therefore, the above distribution function 
$F$ is not NWU. Furthermore, it is easily verified that $F$ is not NBU. Finally, $F$ is none of IFR, DFR, NBU and NWU distributions, although $F$ is concave. Thus, the bounds given in (7) and (8) can not be used for this distribution. However, by Theorem 1, we can get an upper bound on $F^{n *}(t)$ for $t \geq 0$ and $n=1,2, \ldots$. For instance, taking $n=2$ in (11) it is obtained that

$$
F^{2 *}(t) \leq \begin{cases}\frac{2 e^{-2 \sqrt{\frac{t}{2}}}-4 e^{-\sqrt{\frac{t}{2}}}+2}{\left(1-e^{-2}\right)^{2}}, & 0 \leq t<0.6412 \\ \frac{4 e^{-\sqrt{\frac{t}{2}}-2}-2 e^{-2 \sqrt{\frac{t}{2}}}+1-2 e^{-2}-e^{-4}}{\left(1-e^{-2}\right)^{2}}, & 0.6412 \leq t<8 \\ 1, & t \geq 8\end{cases}
$$

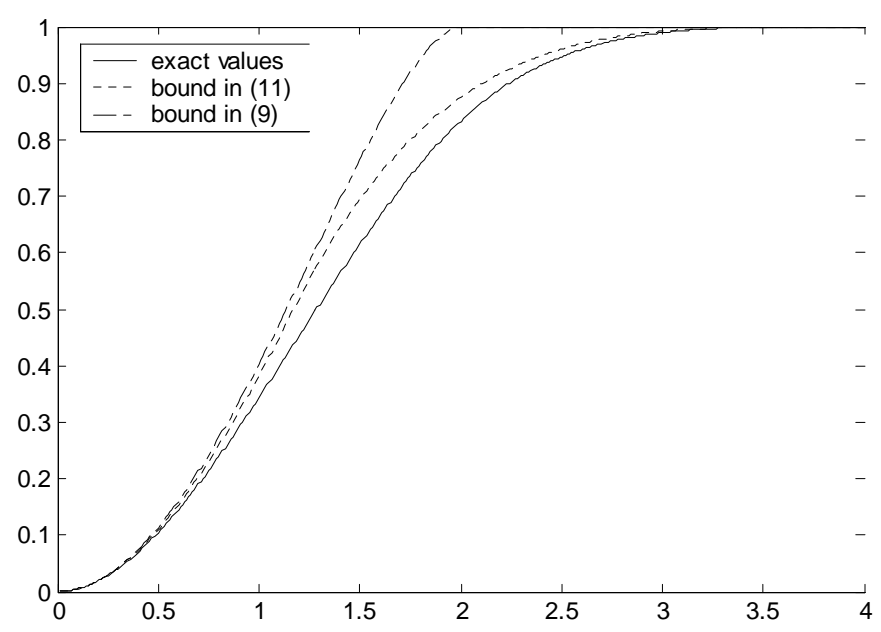

Figure 1. Comparison of the upper bounds for $F^{2 *}(t)$ in Example 2.

We now assume that $F$ is log-concave. An upper bound for $F^{n *}(t)$ in this case is given below.

Theorem 2. If $F$ is continuous and log-concave then for $t \geq 0$ and $n=1,2, \ldots$,

$$
F^{n *}(t) \leq \sum_{j=0}^{n-1} \frac{\left(n K\left(\frac{t}{n}\right)\right)^{j}}{j !} e^{-n K\left(\frac{t}{n}\right)},
$$

where $K(t)=-\ln F(t)$.

Proof. The proof is similar to that of Theorem 1. Let $X_{1}, X_{2}, \ldots, X_{n}$ be independent and identically distributed random variables with distribution function $F$. Since $\ln F$ is concave, choosing $\lambda_{i}=\frac{1}{n}, i=1,2, \ldots, n$ in (5) we have

$$
\ln F\left(\frac{X_{1}+X_{2}+\ldots+X_{n}}{n}\right) \geq \frac{\ln F\left(X_{1}\right)+\ln F\left(X_{2}\right)+\ldots+\ln F\left(X_{n}\right)}{n} .
$$


Then, by (13) and the increasingness of the ln function it is obtained that for $t \geq 0$ and $n=1,2, \ldots$,

$$
\begin{aligned}
F^{n *}(t) & =P\left(X_{1}+X_{2}+\ldots+X_{n} \leq t\right) \\
& =P\left(\ln F\left(\frac{X_{1}+X_{2}+\ldots+X_{n}}{n}\right) \leq \ln F\left(\frac{t}{n}\right)\right) \\
& \leq P\left(\ln F\left(X_{1}\right)+\ln F\left(X_{2}\right)+\ldots+\ln F\left(X_{n}\right) \leq n \ln F\left(\frac{t}{n}\right)\right) \\
& =P\left(-\ln F\left(X_{1}\right)-\ln F\left(X_{2}\right)-\ldots-\ln F\left(X_{n}\right) \geq-n \ln F\left(\frac{t}{n}\right)\right) \\
& =1-F_{Y}\left(n K\left(\frac{t}{n}\right)\right),
\end{aligned}
$$

where $Y=-\ln F\left(X_{1}\right)-\ln F\left(X_{2}\right)-\ldots-\ln F\left(X_{n}\right)$ and $\ln F\left(X_{i}\right)(i=1,2, \ldots, n)$ are independent and identically distributed random variables. Using the probability integral transformation it is easily seen that $Y$ is a gamma distributed random variable with the shape parameter $\alpha=n$ and the scale parameter $\beta=1$. Hence the expression (12) is obtained.

Example 4. Let $F$ be the Weibull distribution function with parameters $\alpha=2$ and $\beta=1$; that is, $F(t)=1-e^{-t^{2}}, t \geq 0$. From Section 1 we have that $F$ is $\log$-concave although $F$ is not concave. Furthermore, it is well known that $F$ is both IFR and NBU. Thus, the upper bound given in (8) or (12) can be used for $F^{n *}(t)$. Let us take $n=2$. Then, the upper bounds in (8) and (12) are obtained as $1-\left(1+t^{2}\right) e^{-t^{2}}$ and $\left(1-e^{-\frac{t^{2}}{4}}\right)^{2}\left(1-2 \ln \left(1-e^{-\frac{t^{2}}{4}}\right)\right)$ respectively. The graphs of these upper bounds are given in Figure 2. Note that an analytical expression of $F^{n *}(t)$ does not exist for $t \geq 0$ and $n=2,3, \ldots$. Now we use a numerical procedure based on the idea of the trapezoidal rule in numerical analysis for computing $F^{n *}(t)$ for given $n$ and $t$ values. The details of this procedure can be found in Aydoğdu [2]. The values of $F^{2 *}(t)$ calculated numerically by this method are also given in Figure 2. These values can be considered as almost exact. From Figure 2, it is seen that the upper bound given in Theorem 2 is better than the upper bound in (8) given by Barlow and Proschan [4]. 


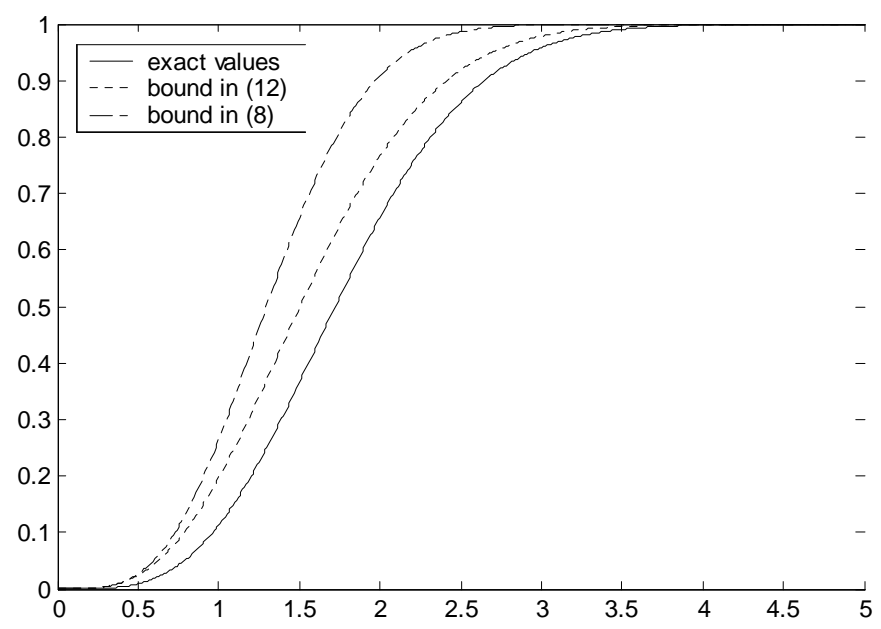

Figure 2. Comparison of the upper bounds for $F^{2 *}(t)$ in Example 4.

Note that the concave distribution functions given in Examples 2 and 3 are also log-concave. Then, the upper bound of Theorem 2 can be used for the $n$-fold convolution of these distribution functions. By Theorem 2, the upper bounds for $F^{2 *}(t)$ are obtained respectively as $\left(\frac{t}{2}-\frac{t^{2}}{16}\right)^{2}\left(1-2 \ln \left(\frac{t}{2}-\frac{t^{2}}{16}\right)\right), 0<t \leq 4$ and $\left(\frac{1-e^{-\sqrt{\frac{t}{2}}}}{1-e^{-2}}\right)^{2}\left(1-2 \ln \left(\frac{1-e^{-\sqrt{\frac{t}{2}}}}{1-e^{-2}}\right)\right), 0<t \leq 8$. Hence, by comparing these upper bounds with the upper bounds given in Examples 2 and 3 it is seen that the upper bounds of Theorem 1 are better than those of Theorem 2. This is not surprising because the concavity of $F$ is a stronger condition than the log-concavity of $F$.

\section{KONKAV VE LOG-KONKAV DAĞILIM FONKSIYYONLARININ n KEZ KONVOLÜSYONU İÇİN BAZI SINIRLAR}

Özet: Genelde bir $F$ dağılım fonksiyonunun kendi kendisiyle olan $n$-kez konvolüsyonu için analitik ifadeler elde etmek mümkün değildir. $F^{n *}$ için sinırların varlığı önemlidir. Bu çalışmada $F$ konkav ya da log-konkav olduğunda olasılık integral dönüşümü yardımıyla $F^{n *}$ için bazı sınırlar verilir.

\section{REFERENCES}

[1] Aydoğdu, H. and Öztürk, F., "Some Monotonicity Properties of Age and Excess Lifetime in Renewal Processes", Jour. of Inst. of Math. and Comp. Sci. (Math. Ser.) Vol.10, No.3 (1997) $145-150$. 
[2] Aydoğdu, H., "A Pointwise Estimator for the k-fold Convolution of a Distribution Function", Commun. Statist. Theor. Meth. 34 (2005) 1939-1956.

[3] Barlow, E. R. and Proschan, F., Mathematical Theory of Reliability. John Wiley \& Sons, Inc., New York (1965).

[4] Barlow, E. R. and Proschan, F., Statistical Theory of Reliability and Life Testing. Holt, Rinehart and Winston, Inc. New York (1981).

[5] Bergstrom, T. and Bagnoli, M., Log-Concave Probability and Its Applications. University of California, Postprints, Paper 11 (2005).

Current address: Ankara University, Faculty of Sciences, Department of Statistics, 06100 Tandoğan-Ankara, Turkey

E-mail address: aydogdu@science.ankara.edu.tr 